\title{
Addition of chemotherapy to intensity-modulated radiotherapy does not improve survival in stage II nasopharyngeal carcinoma patients
}

\author{
Wang Fangzheng ${ }^{1,2, *}$, Jiang Chuner ${ }^{3,}$, Sun Quanquan ${ }^{1,2}$, Ye Zhimin ${ }^{1,2}$, Liu Tongxin ${ }^{1,2}$, Liu Jiping 4 , Masoto \\ Sakamoto ${ }^{5}$, Wu Peng 6 , Shi Kaiyuan7, Qin Weifeng ${ }^{1,2}$, Fu Zhenfu, ${ }^{1,2}$, Jiang Yangming ${ }^{8}$ \\ 1. Department of Radiation Oncology, Zhejiang Cancer Hospital, Zhejiang Hangzhou, 310022, People's Republic of, China \\ 2. Key Laboratory of Radiation Oncology of Zhejiang Province, Zhejiang Hangzhou, 310022, People's Republic of, China \\ 3. Department of Breast Surgery, Zhejiang Cancer Hospital, Zhejiang Hangzhou, 310022, People's Republic of, China \\ 4. Department of Physics, Zhejiang Cancer Hospital, Zhejiang Hangzhou, 310022, People's Republic of, China \\ 5. Department of Radiology, Fukui Red Cross Hospital, Fukui Japan \\ 6. Department of Pathology, Zhejiang Cancer Hospital, Zhejiang Hangzhou, 310022, People's Republic of, China \\ 7. Department of Ultrasonography, Zhejiang Cancer Hospital, Zhejiang Hangzhou, 310022, People's Republic of, China \\ 8. Department of Digital Earth, Institute of Remote Sensing and Digital Earth, CAS, Beijing, 100101, People's Republic of, China \\ *These authors have contributed equally to this work. \\ $\bowtie$ Corresponding author: Jiang Yangming, Email: jym_wm@126.com or Wang Fangzheng, Email: wangfz@zjcc.org.cn \\ (C) Ivyspring International Publisher. This is an open access article distributed under the terms of the Creative Commons Attribution (CC BY-NC) license \\ (https://creativecommons.org/licenses/by-nc/4.0/). See http://ivyspring.com/terms for full terms and conditions.
}

Received: 2018.01.19; Accepted: 2018.02.28; Published: 2018.04.30

\begin{abstract}
In this study, we examined whether combining neoadjuvant chemotherapy (NAC) and/or concurrent chemotherapy (CC) with intensity-modulated radiotherapy (IMRT) improved survival in patients with stage II nasopharyngeal carcinoma (NPC). Two hundred forty-two stage II NPC patients were enrolled between May 2008 and April 2014 and received radical IMRT with simultaneous integrated boost technique using $6 \mathrm{MV}$ photons; some patient groups also received chemotherapy every 3 weeks for 2-3 cycles. The median follow-up duration was 69 months for all patients. At the last follow-up, 18 patients had experienced treatment failure; locoregional relapse among the IMRT alone, NAC+IMRT, $N A C+C C R T$, and CCRT occurred in 3, 3, 4 and 5, respectively; distant metastases in 0,0,2 and 1, respectively, and there was a statistically significant difference among four groups $(P=0.019)$. The 5 -year locoregional relapse-free survival (LRRFS), distant metastasis-free survival (DMFS), progression-free survival (PFS), and overall survival (OS) rates for all patients were $94.7 \%, 98.7 \%, 92.9 \%$, and $93.4 \%$, respectively. Five-year LRRFS, DMFS, PFS, and OS were similar among the IMRT alone, NAC+IMRT, NAC+CCRT, and CCRT treatment groups. Univariate and multivariate analyses revealed that a combined regimen was not an independent prognostic factor for any survival outcome. However, patients who received IMRT plus chemotherapy experienced more acute adverse events than those who received IMRT alone. Thus, the addition of NAC and/or CC to IMRT did not improve survival outcomes, but was associated with higher incidences of acute treatment-associated toxicities than IMRT alone in patients with stage II NPC.
\end{abstract}

Key words: stage II nasopharyngeal carcinoma, intensity-modulated radiotherapy, neoadjuvant chemotherapy, concurrent chemoradiotherapy, toxicity, prognosis

\section{Introduction}

The incidence of nasopharyngeal carcinoma (NPC) varies from 15 to 50 cases per 100,000 annually in Southern China, Singapore, and Malaysia depending on age, ethnicity, and geographical region [1]. Radiotherapy (RT) is the standard treatment for NPC because of its anatomical location and high

radiosensitivity. Intensity-modulated radiation therapy (IMRT) has improved locoregional control, but does little to improve survival outcomes or to prevent distant failure [3, 4]. Meta-analyses of randomized studies indicate that combining RT and chemotherapy reduces the risk of mortality by $18 \%$ 
and increases 5-year survival by $4 \%$ to $6 \%$ [5]. Concurrent chemoradiotherapy (CCRT) with or without adjuvant chemotherapy, which improves overall survival, has become the standard treatment for locoregionally advanced NPC, but is associated with acute toxicities [6-8]. Recent meta-analyses have shown that the addition of neoadjuvant chemotherapy (NAC) to CCRT reduces distant failure in locoregionally advanced NPC patients [9, 10]; another confirmed that NAC followed by CCRT significantly improved progression-free survival (PFS) and overall survival (OS) [11]. These results suggest that addition of NAC and/or concurrent chemotherapy (CC) to IMRT benefits patients with locoregionally advanced NPC. However, it remains unclear whether the addition of chemotherapy to IMRT improves survival in patients with stage II NPC. The American National Comprehensive Cancer Network (NCCN) guidelines nevertheless recommend the same treatment strategy for stage II and stage III/IVA-B NPC.

CCRT prolongs survival outcomes in stage II NPC patients when two-dimensional (2D) RT is used [12-14]; however, IMRT alone might yield similar therapeutic effects and fewer treatment-associated toxicities in these patients compared to combined IMRT and chemotherapy treatments [15-17]. A recent meta-analysis indicated that CCRT improved LRRFS compared to 2D-RT alone, but did not improve survival compared to IMRT alone, in stage II NPC patients [18]. Chen et al. found that CCRT with or without adjuvant chemotherapy (AC) did not improve survival outcomes, but decreased the incidence of acute adverse events, compared to IMRT alone in stage II NPC patients [19]. In contrast, some studies indicate that CCRT did not confer survival benefits in early-stage NPC patients, but increased the occurrence of acute treatment-associated complications, compared to IMRT alone [20-22]. However, a multi-center study demonstrated that CCRT was associated with higher 5-year LRRFS and PFS than IMRT alone in stage II NPC patients [23]. In addition, Luo et al. found that CCRT improved 3-year OS in patients with early-stage NPC [24]. It therefore remains unclear whether combining IMRT with CC improves survival in patients with stage II NPC.

Although few studies that have examined the efficacy of NAC before CCRT or IMRT in stage II NPC, this treatment strategy yielded encouraging outcomes in patients with locoregionally advanced NPC [25-27]. In this retrospective study, we compared the efficacy and toxicities of treatments combining NAC and/or CC with IMRT to IMRT alone in stage II NPC patients.

\section{Materials and Methods}

\section{Patients}

The patients enrolled in this study were hospitalized between May 2008 and April 2014 in the Department of Radiation Oncology, Zhejiang Cancer Hospital. Eligible patients met the following criteria: (i) newly-diagnosed NPC; (ii) stage II; (iii) Eastern Cooperative Oncology Group performance status $\leq 1$; (iv) completion of radical IMRT; (iv) treated with or without chemotherapy, including NAC and/or CC; and (v) no previous anti-cancer treatment. Ultimately, 242 stage II NPC patients were included in this retrospective study, which was approved by the Medical Ethics Committee of Zhejiang Cancer Hospital. All patients provided informed consent.

\section{Baseline examinations}

Patients underwent pretreatment evaluations that included complete histories, physical examinations, hematology and biochemistry profiles, chest radiographs, sonography of the abdomen, bone scans, magnetic resonance images of the nasopharynx, and nasopharyngoscopies. All patients were staged according to the 2010 American Joint Committee on Cancer staging system. Tumor histology was classified per the World Health Organization classification.

\section{Intensity-modulated radiotherapy}

All patients underwent radical IMRT with simultaneous integrated boost technique using $6 \mathrm{MV}$ photons. Thirty-seven patients received IMRT alone. Delineation of target NPC volumes during IMRT treatment was performed as described previously [28-30]. Briefly, gross tumor volumes (GTV) of primary tumors and metastatic lymph nodes were defined as GTVnx and GTVnd and were delineated according to pre- and post-IC MR images, respectively. The clinical target volume of nasopharynx (CTVnx) was defined as GTVnx plus a 7 $\mathrm{mm}$ margin that encompassed the nasopharyngeal mucosa plus $5 \mathrm{~mm}$ submucosal volume. The high-risk clinical target volume (CTV1) included the entire nasopharyngeal cavity, the anterior one- to two-thirds of the clivus, the skull base, the pterygoid plates, the parapharyngeal space, the inferior sphenoid sinus, the posterior one-quarter to one-third of the nasal cavity, and the maxillary sinus and any lymph nodes in drainage pathways containing metastatic lymph nodes. The low-risk clinical target volume (CTV2) included levels IV and $\mathrm{Vb}$ without metastatic cervical lymph nodes.

The PTV was constructed automatically based on each volume with an additional 3-mm margin in 
three dimensions to account for set-up variability. All of the PTVs, including PGTVnx, PTVnx, PTV1, and PTV2, were not delineated outside of the skin surface. Critical normal structures, including the brainstem, spinal cord, parotid glands, optic nerves, chiasm, lens, eyeballs, temporal lobes, temporomandibular joints, mandible, and hypophysis, were contoured and set as OARs during optimization.

The prescribed radiation dose was 69 or $72 \mathrm{~Gy}$ to PGTVnx, 66-70 Gy to PGTVnd, 62-66 Gy to PTVnx, 60-63 Gy to PTV1, and 51-54 Gy to PTV2, delivered in 30 or 33 fractions. Radiation was delivered once daily, five fractions per week, over 6-6.5 weeks for IMRT planning. The dose to OAR was limited on the basis of the RTOG 0225 protocol.

\section{Chemotherapy}

205 patients received platinum-based chemotherapy every three weeks. Of these patients, 25 were treated with CCRT, 48 with NAC followed by IMRT, and 132 with NAC in addition to CCRT. The available NAC regimens included TPF (docetaxel 60 $\mathrm{mg} / \mathrm{m}^{2} /$ day on day 1 , cisplatin $25 \mathrm{mg} / \mathrm{m}^{2} /$ day on days 1 to 3 , and 5 -fluorouracil $500 \mathrm{mg} / \mathrm{m}^{2} /$ day on days 1 to 3 ), TP (docetaxel $60 \mathrm{mg} / \mathrm{m}^{2} /$ day on day 1 , cisplatin $25 \mathrm{mg} / \mathrm{m}^{2} /$ day on days 1 to 3$)$, GP (gemcitabine $1,000 \mathrm{mg} / \mathrm{m}^{2} /$ day on days 1 and 8 , cisplatin $25 \mathrm{mg} / \mathrm{m}^{2} /$ day on days 1 to 3 ), and FP (cisplatin $25 \mathrm{mg} / \mathrm{m}^{2} /$ day on days 1 to 3 , and 5 -fluorouracil $500 \mathrm{mg} / \mathrm{m}^{2} /$ day on days 1 to 3 ). Furthermore, the patients in this study underwent concurrent chemotherapy with cisplatin $\left(80 \mathrm{mg} / \mathrm{m}^{2}\right)$ on 3 days every three weeks for 2-3 cycles.

\section{Efficacy evaluation and follow-up}

Tumor response assessments were performed three times: after the completion of IC, at the end of IMRT, and 3 months after irradiation, which was based on MRI and nasopharynx fiberscope per the Response Evaluation Criteria for Solid Tumors. Systemic chemotherapy adverse events were graded per the National Cancer Institute Common Toxicity Criteria (NCI CTCAE, Version 3.0), and RT-induced toxicities were scored per the Acute and Late Radiation Morbidity Scoring Criteria of the Radiation Therapy Oncology Group.

All subjects underwent weekly examinations for treatment response and toxicities during RT. Follow-ups occurred every 3 months in the first 2 years, every 6 months from the third to the fifth year, and annually thereafter. Each follow-up included careful examination of the nasopharynx and neck nodes by an experienced doctor. MRI scans of the nasopharynx, nasopharynx fiberscope, chest computed tomography radiograph, and ultrasound of abdomen were performed 3 months after the completion of RT and every 6 to 12 months thereafter. Additional examinations were performed as needed to evaluate local relapse or distant metastasis.

\section{Statistical analysis}

The end points of this study included LRRFS, DMFS, PFS, OS, and acute toxicities from IC and CCRT. OS was calculated from the date of enrollment to the date of death or the last follow-up. LRRFS, DMFS, and PFS were calculated from the date of enrollment to the date of locoregional relapse, the occurrence of distant metastasis, diagnosis of disease progression, respectively, or the last follow-up. After recurrence or metastasis, patients were given salvage therapy at their physicians' discretion.

Descriptive statistics were used to compare the patient characteristics and failure modes between the treatment arms. Two independent sample non-parametric tests were used to compare acute toxicities between the treatment arms. Survival curves were generated using the Kaplan-Meier method and compared using log-rank tests. Multivariate analysis was performed using Cox regression models to identify significant prognostic factors. Hazard ratios (HRs) and 95\% confidence intervals (CIs) were calculated for each prognostic factor. IBM SPSS Statistics version 19.0 was used for all data analysis. $P$ $<0.05$ was considered statistically significant.

\section{Results}

\section{Patient characteristics}

Clinical data for newly diagnosed NPC patients who received IMRT at Zhejiang Cancer Hospital between May 2008 and April 2014 were retrospectively reviewed. A total of 242 patients with stage II were enrolled. Basic patient characteristics are summarized in Table 1 . The median age was 50 years (range, 18-77 years), and the male to female ratio was 2.27:1 (168:74). There were no statistically significant differences in age, gender, pathology, $\mathrm{T}$ category, $\mathrm{N}$ category, or clinical subgroup among the four treatment regimens.

\section{Survival}

For all patients, the median follow-up period was 69 months (range, 7-106 months) and the estimated 5-year locoregional relapse-free survival (LRRFS), distant metastasis-free survival (DMFS), progression-free survival (PFS), and overall survival (OS) rates were $94.7 \%, 98.7 \%, 92.9 \%$, and $93.4 \%$, respectively (Fig. 1). Five-year LRRFS, DMFS, PFS, and OS rates in NPC patients with T1N1M0, T2N0M0, and T2N1M0 were $98.6 \%, 87.5 \%$, and $94.6 \%(p=0.111)$, $98.4 \%, 100 \%$, and $97.5 \%(p=0.490)$, and $97.0 \%, 87.5 \%$, 
and $92.2 \%(p=0.223)$, and $95.2 \%, 97.1 \%$, and $91.2 \%$ $(p=0.328)$, respectively; there were no statistically significant differences in these survival outcomes among the three subgroups.

As shown in Figure 2, there were no statistically significant differences among the CCRT, NAC+IMRT, and NAC+CCRT, and IMRT alone groups in 5-year LRRFS $(91.8 \%$ vs. $91.9 \%, p=0.599 ; 95.3 \%$ vs. $91.9 \%$, $p=0.463 ; 95.8 \%$ vs. $91.9 \%, p=0.297 ;)$, DMFS $(100 \%$ vs. $100 \%, \mathrm{p}=1.00 ; 92.5 \%$ vs. $100 \%, \mathrm{p}=0.064 ; 99.2 \%$ vs. $100 \%, \mathrm{p}=0.595)$, PFS (91.8\% vs. $91.9 \%, \mathrm{p}=0.599$; $87.5 \%$ vs. $91.9 \%, p=0.476 ; 95.1 \%$ vs. $91.9 \%, p=0.414)$, or OS $(100 \%$ vs. $97.1 \%, p=0.682 ; 89.0 \%$ vs. $97.1 \%$, $\mathrm{p}=0.228 ; 92.6 \%$ vs. $97.1 \%, \mathrm{p}=0.448)$ rates.

\section{Failure patterns}

Treatment failure occurred in 18 patients (7.5\%) by the last follow-up. Of these patients, 13 (5.3\%) experienced locoregional relapse alone (3 IMRT alone patients, 3 CCRT patients, 2 NAC+IMRT patients, and 5 NAC+CCRT patients), while $5(2.1 \%)$ experienced distant failure alone (four NAC+IMRT patients and one NAC+CCRT patient). Treatment failure information is listed in Table 2.
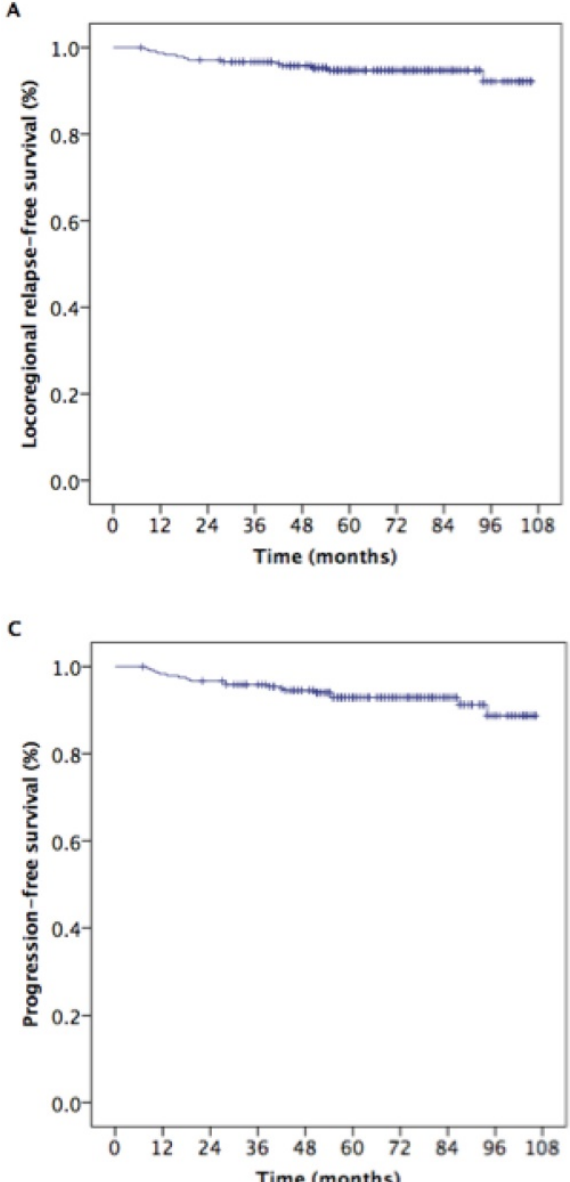

Table 1. Basic characteristics for 242 stage II NPC patients treated with IMRT plus NAC and/or CC.

\begin{tabular}{|c|c|c|c|c|c|c|}
\hline Characteristic & Total & IMRT alone & CCRT & NAC+IMRT & $\mathrm{NAC}+\mathrm{CCRT}$ & $p$ \\
\hline & $\mathrm{N}=242$ & $\mathrm{~N}=37$ & $\mathrm{~N}=25$ & $\mathrm{~N}=48$ & $\mathrm{~N}=132$ & \\
\hline Sex & & & & & & 0.704 \\
\hline Male & 168 & 25 & 15 & 35 & 93 & \\
\hline Female & 74 & 12 & 10 & 13 & 39 & \\
\hline Age (years) & & & & & & 0.711 \\
\hline$<50$ & 109 & 14 & 13 & 21 & 61 & \\
\hline$\geq 50$ & 133 & 23 & 12 & 27 & 71 & \\
\hline WHO pathology & & & & & & 0.998 \\
\hline I & 8 & 1 & 1 & 2 & 4 & \\
\hline II & 16 & 3 & 2 & 3 & 8 & \\
\hline III & 218 & 33 & 22 & 43 & 120 & \\
\hline T stage * & & & & & & 0.535 \\
\hline $\mathrm{T} 1$ & 70 & 13 & 9 & 11 & 37 & \\
\hline $\mathrm{T} 2$ & 172 & 24 & 16 & 37 & 95 & \\
\hline N stage * & & & & & & 0.193 \\
\hline No & 35 & 9 & 4 & 8 & 14 & \\
\hline N1 & 207 & 28 & 21 & 40 & 118 & \\
\hline Clinical stage * & & & & & & 0.219 \\
\hline $\mathrm{T} 1 \mathrm{~N} 1$ & 70 & 13 & 9 & 11 & 37 & \\
\hline $\mathrm{T} 2 \mathrm{NO}$ & 35 & 9 & 4 & 8 & 14 & \\
\hline $\mathrm{T} 2 \mathrm{~N} 1$ & 137 & 15 & 12 & 29 & 81 & \\
\hline
\end{tabular}

Abbreviations: WHO, World Health Organization; IMRT, intensity-modulated radiotherapy; CCRT, concurrent chemoradiotherapy; NAC, neoadjuvant chemotherapy. ${ }^{*}$ The 7 th AJCC/UICC staging system.
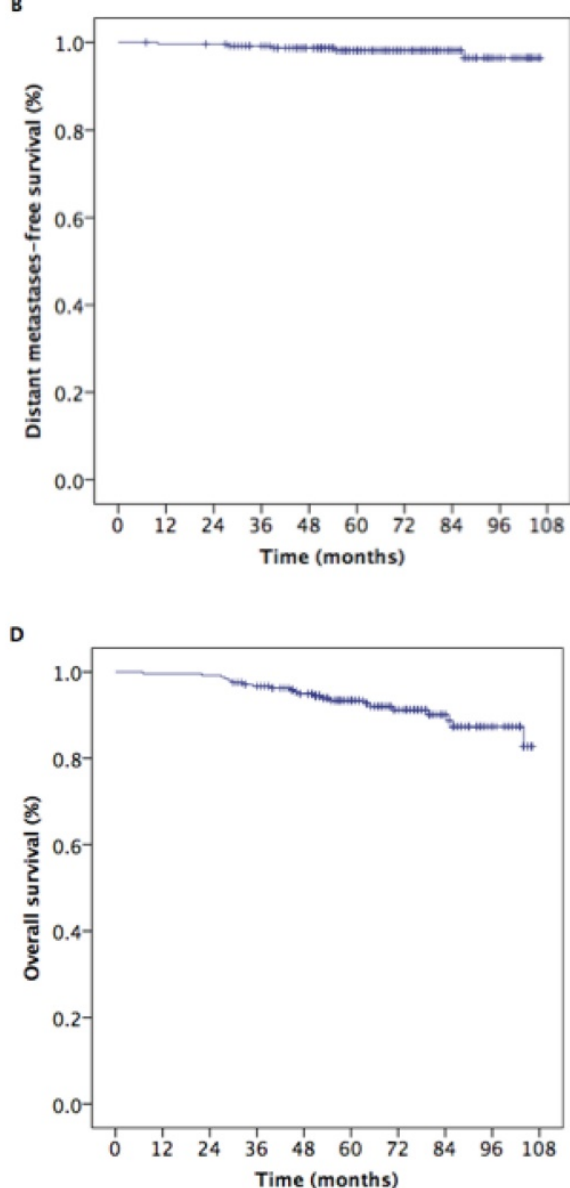

Figure 1. Kaplan-Meier estimates of survival for 242 patients with stage II nasopharyngeal carcinoma. 

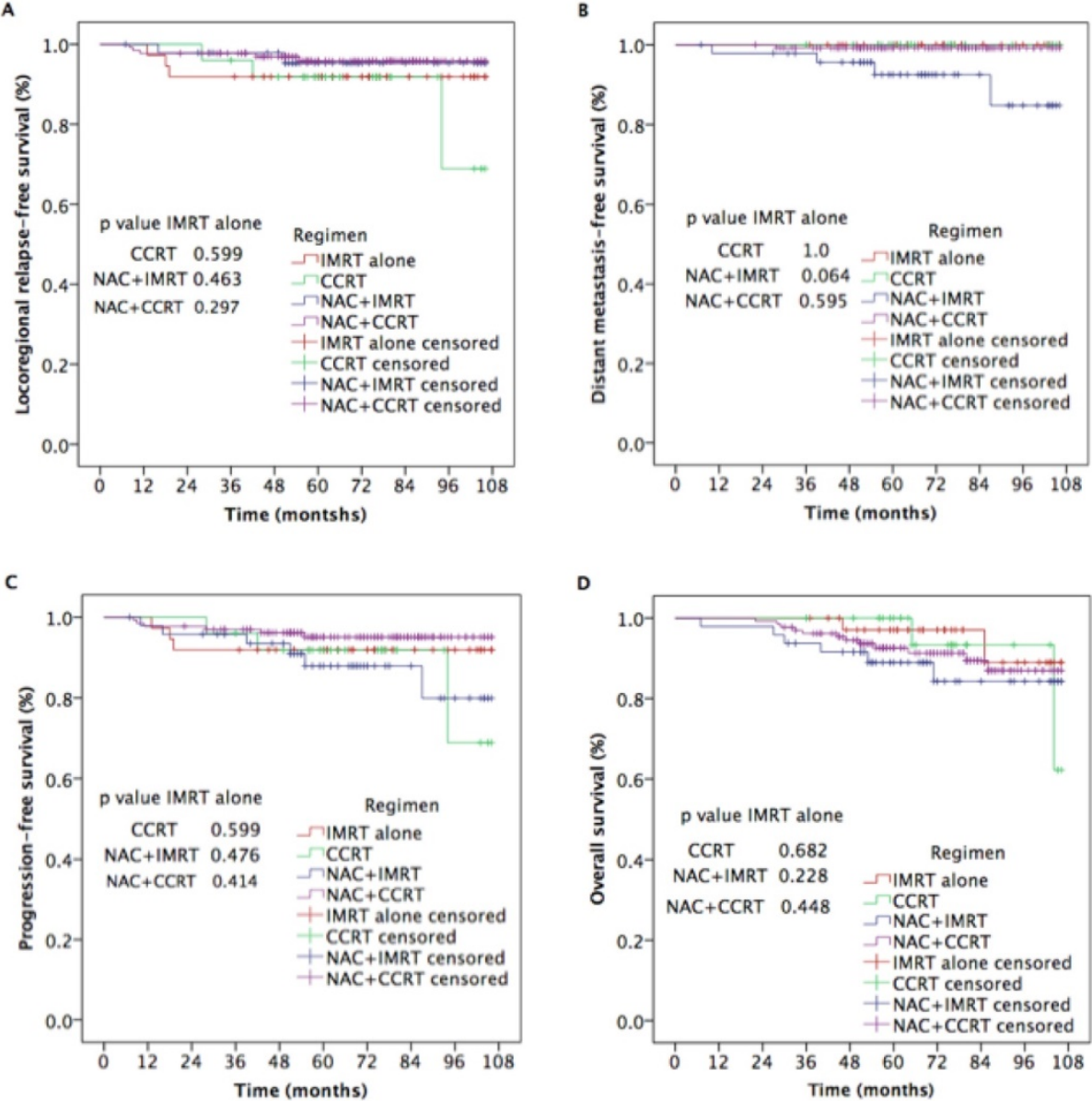

Figure 2. Kaplan-Meier estimates of survival outcomes in nasopharyngeal carcinoma patients receiving additional NAC and/or CC to IMRT and IMRT alone.

Table 2. Treatment failures.

\begin{tabular}{llllll}
\hline Failure mode & $\begin{array}{l}\text { IMRT } \\
\text { alone }\end{array}$ & CCRT & NAC+IMRT & NAC+CCRT & $p$ \\
\cline { 2 - 5 } & $\mathrm{N}=37$ & $\mathrm{~N}=25$ & $\mathrm{~N}=48$ & $\mathrm{~N}=132$ & \\
\hline Locoregional & 3 & 3 & 2 & 5 & 0.019 \\
Distant & 0 & 0 & 4 & 1 & \\
Non-failure & 34 & 22 & 42 & 126 & \\
\hline
\end{tabular}

Abbreviations: IMRT, intensity-modulated radiotherapy, CCRT, concurrent chemoradiotherapy, NAC, neoadjuvant chemotherapy.

\section{Prognostic analysis}

Potential prognostic factors included age, sex, $\mathrm{T}$ category, N category, subgroup, and combined regimen. Univariate and multivariate analyses were used to identify and evaluate the prognostic role of factors that influenced survival outcomes. These analyses, the results of which are shown in Table 3, indicated that there were no differences in any of the survival outcomes among the treatment groups.

\section{Subgroup analysis}

We evaluated treatment efficacy in 137 NPC patients with T2N1M0. Five-year LRRFS, DMFS, PFS, and OS rates in these patients were $94.6 \%, 97.5 \%$, $92.2 \%$, and 91.6\%, respectively (Figure 3).
Furthermore, as shown in Figure 4, there were no statistically significant differences in 5-year LRRFS $(86.5 \%, 91.7 \%, 96.4 \%, 95.8 \%, p=0.217)$, DMFS $(100 \%$, $100 \%, 91.6 \%, 98.8 \%, p=0.058)$, PFS $(86.7 \%, 91.7 \%$, $88.0 \%, 94.6 \%, p=0.302)$, or OS $(92.9 \%, 100 \%, 89.7 \%$, $90.7 \%, p=0.931$ ) among the four treatment regimens.

Table 3. Prognostic factors for survival outcomes from univariate and multivariate analyses.

\begin{tabular}{|c|c|c|c|c|c|c|c|c|c|}
\hline \multirow[t]{3}{*}{ Variate } & \multirow[t]{3}{*}{ Category } & \multicolumn{8}{|l|}{$p$ value } \\
\hline & & \multicolumn{4}{|c|}{ Univariate analysis } & \multicolumn{4}{|c|}{ Multivariate analysis } \\
\hline & & LRRFS & DMFS & PFS & OS & LRRFS & DMFS & PFS & OS \\
\hline \multirow{2}{*}{$\begin{array}{l}\text { Age } \\
\text { (years) }\end{array}$} & $<50$ & Ref & Ref & Ref & Ref & Ref & Ref & Ref & Ref \\
\hline & $\geq 50$ & 0.103 & 0.781 & 0.118 & 0.177 & 0.097 & 0.851 & 0.106 & 0.185 \\
\hline \multirow[t]{2}{*}{ Sex } & Male & Ref & Ref & Ref & Ref & Ref & Ref & Ref & Ref \\
\hline & Female & 0.963 & 0.654 & 0.860 & 0.873 & 0.871 & 0.615 & 0.736 & 0.777 \\
\hline \multirow[t]{2}{*}{ T stage ${ }^{*}$} & $\mathrm{~T} 1$ & Ref & Ref & Ref & Ref & Ref & Ref & Ref & Ref \\
\hline & $\mathrm{T} 2$ & 0.093 & 0.665 & 0.095 & 0.319 & 0.121 & 0.742 & 0.114 & 0.317 \\
\hline \multirow[t]{2}{*}{ N stage* } & No & Ref & Ref & Ref & Ref & Ref & Ref & Ref & Ref \\
\hline & $\mathrm{N} 1$ & 0.098 & 0.340 & 0.375 & 0.418 & 0.477 & 0.972 & 0.850 & 0.387 \\
\hline \multirow[t]{4}{*}{ Regimen } & IMRT alone & Ref & Ref & Ref & Ref & Ref & Ref & Ref & Ref \\
\hline & CCRT & 0.599 & 1.0 & 0.599 & 0.682 & 0.432 & 0.988 & 0.471 & 0.569 \\
\hline & NAC+IMRT & 0.463 & 0.064 & 0.476 & 0.228 & 0.067 & 0.990 & 0.113 & 0.924 \\
\hline & $\mathrm{NAC}+\mathrm{CCRT}$ & 0.297 & 0.595 & 0.414 & 0.448 & 0.982 & 0.028 & 0.082 & 0.492 \\
\hline
\end{tabular}



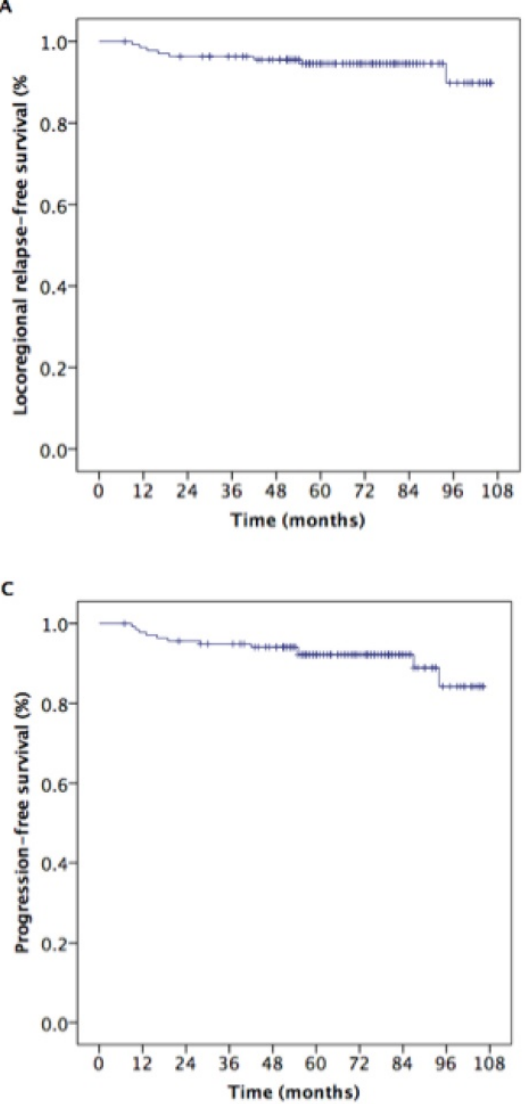
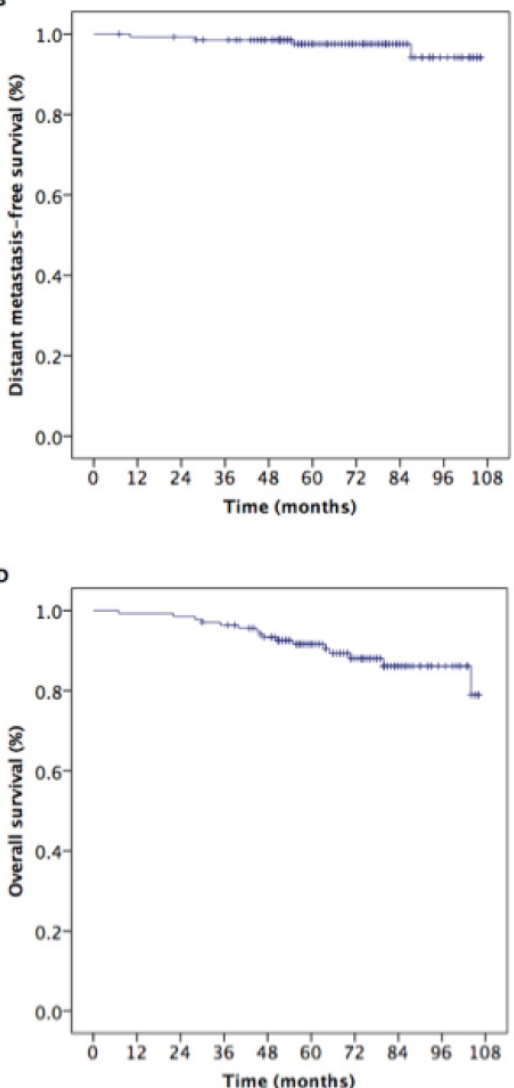

Figure 3. Kaplan-Meier estimates of survival outcomes in 137 nasopharyngeal carcinoma patients with T2N1.
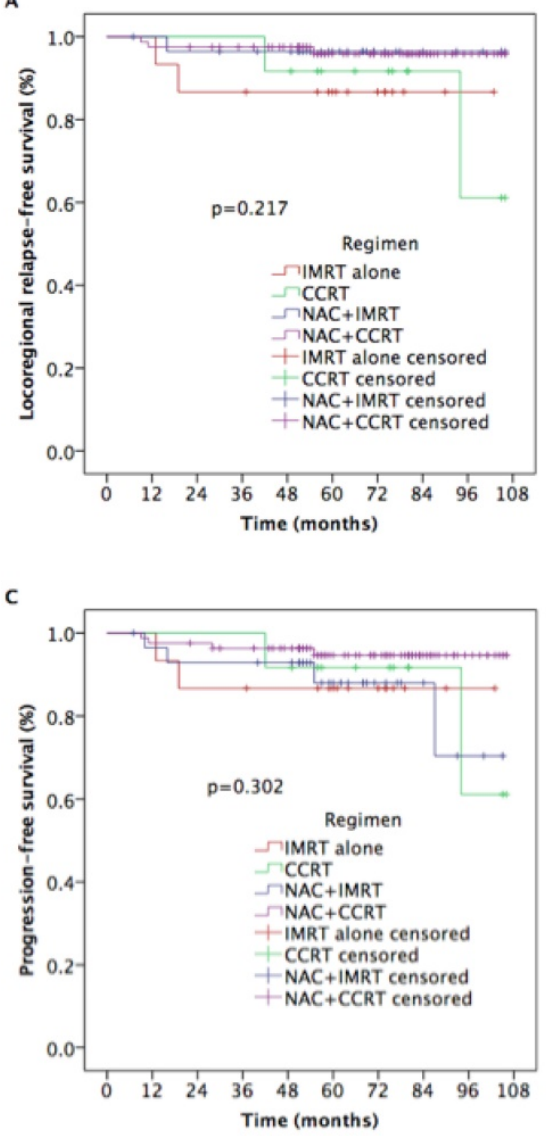
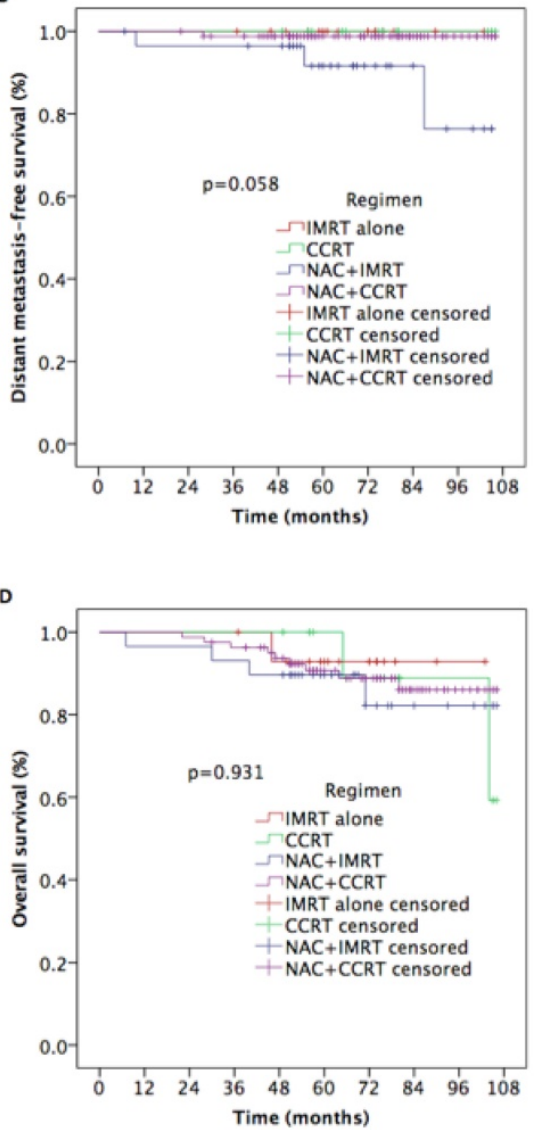

Figure 4. Kaplan-Meier estimates of survival outcomes in $137 \mathrm{~T} 2 \mathrm{~N} 1$ nasopharyngeal carcinoma patients receiving IMRT with or without chemotherapy. 


\section{Safety and toxicity}

The most commonly observed complications included hematologic and non-hematologic side effects. During the period of treatment (Table 4), incidences of grade 3-4 leukocytopenia and neutropenia were higher in patients treated with both NAC and IMRT or CCRT compared to those treated with IMRT alone $(p=0.033$ and $p=0.048)$. In addition, incidence of grade 3-4 mucositis was higher in patients treated with NAC and/or CC as well as IMRT than in those treated with IMRT alone $(p=0.012)$. There were no other significant differences in treatment toxicity among the four arms.

Table 4. Acute treatment-associated toxicities for the four regimens.

\begin{tabular}{lcccccccccc}
\hline Adverse events & \multicolumn{1}{c}{ IMRT alone } & \multicolumn{1}{c}{ CCRT } & \multicolumn{3}{c}{ NAC+IMRT } & NAC+CCRT & $p$ \\
\cline { 2 - 9 } & $0-2$ & $3-4$ & $0-2$ & $3-4$ & $0-2$ & $3-4$ & $0-2$ & $3-4$ & \\
\hline Hematologic & & & & & & & & & \\
Leukocytopenia & 35 & 2 & 20 & 5 & 35 & 13 & 95 & 37 & 0.033 \\
Neutropenia & 34 & 3 & 18 & 7 & 33 & 15 & 92 & 40 & 0.048 \\
Anemia & 36 & 1 & 22 & 3 & 44 & 4 & 127 & 5 & 0.245 \\
Thrombocytopenia & 37 & 0 & 23 & 2 & 41 & 7 & 119 & 13 & 0.134 \\
Liver function & 37 & 0 & 24 & 1 & 45 & 3 & 125 & 7 & 0.517 \\
Renal function & 37 & 0 & 25 & 0 & 47 & 1 & 128 & 4 & 0.590 \\
Non-hematologic & & & & & & & & & \\
Mucositis & 36 & 1 & 20 & 5 & 45 & 3 & 105 & 27 & 0.012 \\
Dermatitis & 37 & 0 & 23 & 2 & 45 & 3 & 125 & 7 & 0.453 \\
Diarrhea & 37 & 0 & 25 & 0 & 46 & 2 & 128 & 4 & 0.511 \\
Nausea/vomiting & 37 & 0 & 24 & 1 & 42 & 6 & 118 & 14 & 0.127 \\
\hline
\end{tabular}

Abbreviations: IMRT, intensity-modulated radiotherapy; CCRT, concurrent chemoradiotherapy; NAC, neoadjuvant chemotherapy.

\section{Discussion}

In this study, we found that combining NAC and/or CC with IMRT treatment did not improve survival outcomes in stage II NPC patients, but did increase incidences of acute treatment-associated toxicities, compared to treatment with IMRT alone. Furthermore, in subgroup analysis, IMRT was similarly effective whether or not it was combined with chemotherapy in patients in the T2N1M0 subgroup. The addition of NAC and/or CC to IMRT therefore did not seem to benefit this patient subgroup.

Although the same treatments are recommended for stage II and stage III-IVB NPC, few studies have examined the efficacy of different treatments in stage II NPC patients, and the effects of NAC and/or CC treatment in these patients remain controversial. In the only existing phase III randomized trial, combining $2 \mathrm{D}$ RT with CC improved 5-year OS, PFS, and DMFS compared to 2D RT alone [14]. Similar results were obtained in several retrospective studies $[12,13,24]$. However, other studies indicate that IMRT treatment alone results in high survival rates in stage II NPC patients $[15,17]$. Su et al. found that outcomes were similar after CCRT or IMRT alone in stage II NPC patients in a retrospective study [20]. In a propensity score matching study of 482 patients with low-risk NPC, 4-year OS, DMFS, and LRFS were also similar after treatment with CCRT or IMRT alone [21]. Chen et al. compared the outcomes of CCRT with or without AC and IMRT alone in stage II NPC, and observed that 5-year OS, LRRFS, DMFS, and PFS were similar among the three arms [19]. In contrast, two studies have found that CCRT improves survival outcomes in stage II NPC patients $[23,24]$.

Chua et al. found that NAC improved 5-year OS and DMFS in NPC patients in the T1-2N0-1 subgroup [31]. However, Song et al. found that NAC with cisplatin and 5-fluorouracil before IMRT did not prolong DMFS or OS [32]. Kang and Tam also found that adding NAC to IMRT did not improve any survival outcomes in NPC patients [15,24]. Thus, it remains unclear whether combining chemotherapy with RT improves survival outcomes in stage II NPC. However, a recent meta-analysis demonstrated that CCRT resulted in longer LRRFS than RT alone when 2D RT was used to treat stage II NPC, while IMRT alone resulted in similar survival outcomes and lower frequencies of acute toxicities than CCRT [18].

In this study, we examined survival over a long follow-up time; 5-year LRRFS, DMFS, PFS, and OS rates for stage II NPC patients were $94.6 \%, 97.5 \%$, $92.2 \%$ and $91.6 \%$, respectively. However, there were no statistically significant differences in 5-year LRRFS, DMFS, PFS, or OS rates when NAC and/or CC were combined with IMRT compared to IMRT treatment alone. In addition, univariate and multivariate analyses indicated that the addition of NAC and/or CC did not improve survival outcomes for these patient subgroups.

Patients with T2N1M0 disease are at high risk of distant failure $[14,17,23]$. Guo et al. found that OS and DMFS were poorer in T2N1M0 NPC patients than in T1N1M0 patients [33]. In contrast, we observed here that 5-year LRRFS, DMFS, PFS, and OS rates were similar among patients with T1N1M0, T2N0M0, and T2N1M0 disease. Moreover, subgroup analysis of 137 patients with T2N1M0 disease revealed no differences in 5-year LRRFS, DMFS, PFS, or OS among the four treatment regimens.

Some limitations of this study should be considered when interpreting the results. First, this retrospective study involved patients from a single center who received various chemotherapy regimens. Second, only acute treatment-associated toxicities were evaluated here; late-stage complications were not considered. Third, acute toxicities were assessed based on medical record information alone. Finally, 
the sample sizes for individual treatment arms were relatively small. These results should therefore be regarded as preliminary, and additional prospective, randomized, large-sample, multi-center phase III clinical trials should be conducted to confirm our findings. In summary, the addition of NAC and/or CC to IMRT was not associated with any survival benefits in stage II NPC patients; IMRT alone may therefore be the best treatment option for these patients.

\section{Acknowledgements}

This study was supported by grants from the Medical Science Foundation of Zhejiang Health Bureau (No. 2013KYB033, No. 2009B026, No. 2006A016, No. 2005B012, No. 2004B014) and the National Natural Science Foundation of China (No. 81502646, No. 81502647).

\section{Competing Interests}

The authors have declared that no competing interest exists.

\section{References}

1. Tang LL, Chen WQ, Xue WQ, et al. Global trends in incidence and mortality of nasopharyngeal carcinoma. Cancer Letters 2016; 374: 22-30.

2. Chen L, Mao YP, Xie FY, et al. The seventh edition of UICC/AJCC staging system for nasopharyngeal carcinoma is prognostically useful for patients treated with intensity-modulated radiotherapy from an endemic area in China. Radiother Oncol 2012; 104: 331-337.

3. Wee J. Nasopharyngeal carcinoma: a promising future. Lancet Oncol 2012; 13 : 116-118.

4. Lai SZ, Li WF, Chen L, et al. How does intensity modulated radiotherapy versus conventional two-dimensional radiotherapy influence the treatment results in nasopharyngeal carcinoma patients? Int J Radiat Oncol Biol Phys 2011; 80: 661-668.

5. Al-Sarraf M and Reddy MS. Nasopharyngeal carcinoma. Curr Treat Options Oncol 2002; 3: 21-32

6. Al-Sarraf $\mathrm{M}$, LeBlanc $\mathrm{M}$, Giri PG, et al. Chemoradiotherapy versus radiotherapy in patients with advanced nasopharyngeal cancer: phase III randomized Intergroup study 0099. J Clin Oncol 1998; 16: 1310-1317.

7. Lee AW, Tung SY, Chua DT, et al. Randomized trial of radiotherapy plus concurrent-adjuvant chemotherapy vs radiotherapy alone for regionally advanced nasopharyngeal carcinoma. J Natl Cancer Inst 2010; 102: 1188-1198.

8. Baujat B, Audry H, Bourhis J, et al. Chemotherapy in locally advanced nasopharyngeal carcinoma: an individual patient data meta-analysis of eight randomized trials and 1753 patients. Int J Radiat Oncol Biol Phys 2006; 64: 47-56

9. OuYang PY, Xie C, Mao YP, et al. Significant efficacies of neoadjuvant chemotherapy and adjuvant chemotherapy for nasopharyngeal carcinoma by meta-analysis of published literature-based randomized, control trials. Ann Oncol 2013, 24:2136-2146.

10. Chen YP, Guo R, Liu N, et al. Efficacy of the additional neoadjuvant chemotherapy to concurrent chemoradiotherapy for patients with locoregionally advanced nasopharyngeal carcinoma: a Bayesian network meta-analysis of randomized controlled trials. J Cancer 2015; 6: 883-892.

11. Wang MM, Tian HM, Li G, et al. Significant benefits of adding neoadjuvant chemotherapy before chemoradiotherapy for locoregionally advanced nasopharyngeal carcinoma: a meta-analysis of randomized controlled trials. Oncotarget 2016; 7: 48375-390.

12. Cheng SH, Tsai SY, Yen KL, et al. Concomitant radiotherapy and chemotherapy for early-stage nasopharyngeal carcinoma. J Clin Oncol, 2000; 18(10): 2040-2045.

13. $\mathrm{Xu} \mathrm{T}$, Hu C, Wang $\mathrm{X}$, Shen $\mathrm{C}$. Role of chemoradiotherapy in intermediate prognosis nasopharyngeal carcinoma. Oral Oncology, 2011; 47(5): 408-413.

14. Chen QY, Wen YF, Guo L, et al. Concurrent chemotherapy vs radiotherapy alone in stage II nasopharyngeal carcinoma: phase III randomized trial. J Natl Cancer Inst, 2011, 103(23): 1761-1770.

15. Tham IW, Lin S, Pan J, et al. Intensity-modulated radiateon therapy without concurrent chemotherapy for stage IIb nasopharyngeal cancer. Am J Clin Oncol, 2010; 33(3): 294-299.
16. Lai SZ, Li WF, Chen L, et al. How does intensity-modulated radiotherapy versus conventional two-dimensional radiotherapy influence the treatment results in nasopharyngeal carcinoma Patients? Int J Radiat Oncol Biol Phys, 2011; 80(3): 661-668.

17. Su SF, Han F, Zhao C, et al. Long-term outcomes of early-stage nasopharyngeal carcinoma patients treated with intensity-modulated radiotherapy alone. Int J Radiat Oncol Biol Phys, 2012; 82(1): 327-333.

18. Xu C, Zhang LH, Chen YP, et al. Chemoradiotherapy versus radiotherapy alone in stage II nasopharyngeal carcinoma: a systemic review and meta-analysis of 2138 patients. J Cancer, 2017; 8(20): 287-297.

19. Chen KH, Zhu XD, Li L, et al. Comparison of the efficacy between concurrent chemoradiotherapy with or without adjuvant chemotherapy and intensity-modulated radiotherapy alone for stage II nasopharyngeal carcinoma. Oncotarget 2016; 7(41):69041-69050.

20. Su Z, Mao YP, Tang J, et al. Long-term outcomes of concurrent chemoradiotherapy versusradiotherapy alone in stage II nasopharyngeal carcinoma treated with IMRT: a retrospective study. Tumour Biol, 2016; 37(4):4429-4438.

21. Zhang LN, Gao YH, Lan XW, et al. Propensity score matching analysis of cisplatin-based concurrent chemotherapy in low risk nasopharyngeal carcinoma in the intensity-modulated radiotherapy era. Oncotarget, 2015, 6(4): 44019-44029.

22. Xu T, Shen $\mathrm{C}, \mathrm{Zhu}$ g, Hu C. Omission of chemotherapy in early stage nasopharyngeal carcinoma treated with IMRT: a paired cohort study. Medicine (Baltimore) 2015, 94(39): e1457.

23. Luo S, Zhao L, Wang J, et al. Clinical outcomes for early-stage nasopharyngeal carcinoma with predominantly WHO II histology treated by intensity -modulated radiation therapy with or without chemotherapy in nonendemic region of china. Head Neck, 2014; 36(6): 841-847.

24. Kang MK, Oh D, Cho KH, et al. Role of chemotherapy in stage II nasopharyngeal carcinoma treated with curative radiotherapy. Cancer Res Treat, 2015; 47(4): 871-878.

25. Sun Y, Li WF, Chen NY, et al. Induction chemotherapy plus concurrent chemotherapy versus concurrent chemoradiotheray alone in locoregionally advanced nasopharyngeal carcinoma: a phase 3, multicentre, randomized controlled trial. Lancet Oncol. 2016, 12(17): 1509-1520.

26. Wang FZ, Sun $Q Q$, Jiang $C E$, et al. Gemcitabine/cisplatin induction chemotherapy before concurrent chemotherapy and intensity-modulated radiotherapy improves outcomes for locoregionally advanced nasopharyngeal carcinoma. Oncotarget, 2017; 8(57): 96798-96808.

27. Qiu WZ, Huang PY, Shi JL, et al. Neoadjuvant chemotherapy plus intensity-modulated radiotheapy versus concurrent chemoradiotherapy plus adjuvant chemotherapy for the treatment of locoregionally advanced nasopharyngeal carcinoma: a retrospective controlled study. Chin J Cancer, 2016 Jan 6; 35:2. doi: 10.1186/s40880-015-0076-9.

28. Wang FZ, Jiang CE, Wang L, et al. Addition of 5-fluorouracil to first-line induction chemotherapy with docetaxel and cisplatin before concurrent chemoradiotherapy does not improve survival in locoregionally advanced nasopharyngeal carcinoma. Oncotarget 2017, 8 (53): 91150-91161.

29. Wang FZ, Jiang CE, Ye ZM, et al. Efficacy and safety of nimotuzumab with neoadjuvant chemotherapy followed by concurrent chemoradiotherapy for locoregionally advanced nasopharyngeal carcinoma. Oncotarget, 2017, 8(43): 75544-75556

30. Wang FZ, Jiang CE, Wang L, et al. Outcome and long-term efficacy of four facio-cervical fields conformal radiotherapy for nasopharyngeal carcinoma. Oncotarget, 2017; 8(24): 39756-39765.

31. Chua DT, Ma J, Sham JS, et al. Improvement of survival after addition of induction chemotherapy to radiotherapy in patients with early-stage nasopharyngeal carcinoma: subgroup analysis of two phase III trials. Int J Radiat Oncol Biol Phys. 2006;65(5):1300-1306.

32. Song $\mathrm{CH}, \mathrm{Wu} \mathrm{HG}, \mathrm{He} \mathrm{DS}$, et al. Treatment outcomes for radiotherapy alone are comparable with neoadjuvant chemotherapy followed by radiotherapy in early-stage nasopharyngeal carcinoma. Laryngoscope. 2008;118:663-70.

33. Guo $\mathrm{Q}, \mathrm{Lu} \mathrm{T}$, Lin $\mathrm{S}$, et al. Long-term survival of nasopharyngeal carcinoma patients with Stage II in intensity-modulated radiation therapy era. Jpn J Clin Oncol. 2016; 46(3): 241-7. 